\title{
Fertility concerns of the transgender patient
}

\author{
Philip J. Cheng ${ }^{1}$, Alexander W. Pastuszak ${ }^{1}$, Jeremy B. Myers ${ }^{1}$, Isak A. Goodwin ${ }^{2}$, James M. Hotaling ${ }^{1,3}$ \\ ${ }^{1}$ Division of Urology, Department of Surgery, ${ }^{2}$ Division of Plastic Surgery, Department of Surgery, ${ }^{3}$ Department of Obstetrics \& Gynecology, \\ University of Utah, Salt Lake City, UT, USA \\ Contributions: (I) Conception and design: PJ Cheng, JM Hotaling; (II) Administrative support: None; (III) Provision of study materials or patients: \\ None; (IV) Collection and assembly of data: PJ Cheng; (V) Data analysis and interpretation: None; (VI) Manuscript writing: All authors; (VII) Final \\ approval of manuscript: All authors. \\ Correspondence to: James M. Hotaling, MD, MS, FECSM. Department of Surgery (Urology), University of Utah, Suite 201, 675 Arapeen, Salt Lake \\ City, UT 84108, USA. Email: jim.hotaling@hsc.utah.edu.
}

\begin{abstract}
Transgender individuals who undergo gender-affirming medical or surgical therapies are at risk for infertility. Suppression of puberty with gonadotropin-releasing hormone agonist analogs (GnRHa) in the pediatric transgender patient can pause the maturation of germ cells, and thus, affect fertility potential. Testosterone therapy in transgender men can suppress ovulation and alter ovarian histology, while estrogen therapy in transgender women can lead to impaired spermatogenesis and testicular atrophy. The effect of hormone therapy on fertility is potentially reversible, but the extent is unclear. Gender-affirming surgery (GAS) that includes hysterectomy and oophorectomy in transmen or orchiectomy in transwomen results in permanent sterility. It is recommended that clinicians counsel transgender patients on fertility preservation (FP) options prior to initiation of gender-affirming therapy. Transmen can choose to undergo cryopreservation of oocytes or embryos, which requires hormonal stimulation for egg retrieval. Uterus preservation allows transmen to gestate if desired. For transwomen, the option for FP is cryopreservation of sperm either through masturbation or testicular sperm extraction. Experimental and future options may include cryopreservation and in vitro maturation of ovarian or testicular tissue, which could provide prepubertal transgender youth an option for FP since they lack mature gametes. Successful uterus transplantation with subsequent live birth is a new medical breakthrough for cisgender women with uterus factor infertility. Although it has not yet been performed in transgender women, uterus transplantation is a potential solution for those who wish to get pregnant. The transgender population faces many barriers to care, such as provider discrimination, lack of information, legal barriers, scarcity of fertility centers, financial burden, and emotional cost. Further research is necessary to investigate the feasibility of experimental FP options, provide better evidence-based information to clinicians and transgender patients alike, and to improve access to and quality of reproductive services for the transgender population.
\end{abstract}

Keywords: Cryopreservation; fertility; transgender; uterus transplantation

Submitted Nov 21, 2018. Accepted for publication May 20, 2019.

doi: $10.21037 /$ tau.2019.05.09

View this article at: http://dx.doi.org/10.21037/tau.2019.05.09

\section{Introduction}

There are an estimated 1.4 million transgender individuals in the United States, representing $0.6 \%$ of the population (1). The term "transgender" describes a person whose gender identity is incongruent with the phenotypic sex assigned at birth. Transgender people may choose to transition from female to male (transgender man, transman, or FTM) or male to female (transgender woman, transwoman, or MTF).

The gender affirmation process may include emotional, social, medical, and surgical aspects of transition, though not all transgender people desire medical or surgical intervention $(2,3)$. Some transgender individuals undergo gender-affirming interventions to masculinize or feminize 
Table 1 Fertility preservation options for transgender people

\begin{tabular}{lll}
\hline Age group & Transgender men (assigned female at birth) & Transgender women (assigned male at birth) \\
\hline Pre-pubertal & Ovarian tissue cryopreservation ${ }^{\dagger}$ & Testicular tissue cryopreservation \\
Post-pubertal & Oocyte cryopreservation & Sperm cryopreservation \\
& Embryo cryopreservation (with partner's or donor's sperm) & Embryo cryopreservation (with partner's or donor's egg) \\
& Uterus preservation & Uterus transplantation $^{\ddagger}$ \\
\hline
\end{tabular}

${ }^{\dagger}$, experimental; ${ }^{\ddagger}$, theoretical.

body parts to bring their physical appearance more in line with their gender identity. According to the 2015 United States Transgender Survey (USTS), which surveyed 27,715 respondents, $49 \%$ had received gender-affirming hormone therapy (GAHT), while $25 \%$ had undergone some form of gender-affirming surgery (GAS) (4). A minority of transgender individuals had undergone gender-affirming bottom surgery, such as hysterectomy (14\%), metoidioplasty $(2 \%)$, and phalloplasty (3\%) for transmen and vaginoplasty/ labiaplasty (12\%) and orchiectomy (11\%) for transwomen (4). Both transgender men and women are at risk of losing their reproductive potential during the process of medical or surgical transition with GAHT or gender-affirming bottom surgery. For instance, transmen who undergo hysterectomy and oophorectomy and transwomen who undergo orchiectomy are rendered permanently sterile. Accordingly, the World Professional Association for Transgender Health (WPATH), the Endocrine Society, the American Society for Reproductive Medicine (ASRM), and the European Society of Human Reproduction and Embryology (ESHRE) recommend that clinicians counsel their transgender patients on the potential for reduced fertility and fertility preservation (FP) options (Table 1) prior to administering puberty-suppressing medications or GAHT $(2,3,5,6)$.

Urologists, gynecologists, and plastic surgeons are usually the primary surgeons performing gender-affirming bottom surgeries. Unlike many professional organizations, the American Urological Association (AUA) does not have an official statement on transgender care or guidelines for the application of gender-affirming hormone therapy or surgery. Nonetheless, it is important for urologists to inform their patients of the fertility risks of undergoing GAS and educate them on their options. The purpose of this article is to review the different fertility concerns that transgender individuals face and examine the current and potential future options for fertility preservation while also providing clinicians a framework for educating transgender patients.

\section{Fertility desires for transgender individuals}

There is a widespread assumption that transgender people do not want to have biological children (7). On the contrary, several studies have shown that many transgender individuals want biological children (7-11). One study of 50 transmen showed that $54 \%$ desired children (12). Similarly, in a survey of 121 transwomen, $51 \%$ would have strongly considered or undergone sperm cryopreservation if they had been given the option by a provider (13). A review of 51 studies examining the prevalence and characteristics of transgender parents showed that in the majority of studies, $25-50 \%$ of transgender people reported being parents (14). People who "come out" or transition later in life tend to have higher parenting rates than those who transitioned at younger ages, likely due to individuals having children with a partner before identifying as transgender (14). Now that acceptance of the transgender population has become more widespread and gender-affirming treatment is increasingly covered by insurance, transgender individuals have started to seek treatment at a younger age, when reproductive wishes may not yet be clearly defined and many may wish to have children after transition $(5,6,12,15)$.

Though a few studies have shown there is a strong desire by transgender adults to have biological children, that desire seems to be less pronounced among transgender youth. In a study by Nahata et al. examining 73 post-pubertal transgender patients who presented to a pediatric endocrinology clinic for hormone therapy, 72 had documented fertility counseling prior to initiation of hormone therapy, though only 2 patients attempted FP (16). Of these 73 patients, $45 \%$ mentioned a desire or plan to adopt, while $21 \%$ said they never wanted to have children (16).

Some studies have shown that individuals that identify as lesbian, gay, bisexual, transgender, and queer (LGBTQ) are more open to alternative methods of family building compared with heterosexuals. In a study comparing adolescent and young adult LGBTQ and heterosexual 
cancer survivors, LGBTQ individuals were more open to raising non-biological children or to not having children than heterosexuals (17). Another study of 156 transgender and gender-nonconforming adolescents showed that $70.5 \%$ were interested in adoption, while only $35.9 \%$ were interested in biological parenthood (18). Of note, only $20.5 \%$ had discussed fertility with a healthcare provider and only $13.5 \%$ discussed effects of hormones on fertility. Another study by Chen et al. of 105 transgender adolescents who presented to a pediatric gender clinic, only 13 (12.4\%) were seen in formal consultation for FP before initiating hormones, 5 of which utilized FP services (19).

A possible explanation for the low utilization of $\mathrm{FP}$ among transgender youth could be that they do not want to delay medical transition (19). Other barriers include the cost and invasiveness of procedures. It is possible that transgender youth may change their perspectives about FP later in life, which makes it all the more important to counsel these patients effectively before initiating treatment that could have potentially irreversible effects on fertility.

\section{Effects of hormone therapy on fertility}

\section{Effects of puberty suppression}

Pubertal suppression with gonadotropin-releasing hormone agonist analogs $(\mathrm{GnRHa})$ is used in the pediatric transgender population as early as Tanner stage 2 . This treatment prevents the development of permanent secondary sex characteristics incongruent with gender identity and can alleviate the psychological distress associated with these changes (20). Furthermore, it provides more time for these children to explore their gender identity. GnRHa-based pubertal suppression is reversible, but it also pauses maturation of germ cells, which could affect fertility potential (21-23). In children treated with GnRHa, 43 of 49 patients had a decrease in testicular volume (24). Similarly, a study of 87 girls with precocious puberty while on GnRHa showed a decrease in ovarian and uterine size during treatment, which subsequently increased in size with resumption of menstruation approximately 1 year after discontinuing therapy (25).

\section{Effects of testosterone therapy}

Gender-affirming hormone therapy in transgender individuals may impact gonadal function and the longterm effects on future fertility are unknown. In transmen, testosterone induces amenorrhea by suppressing ovulation. There are several studies that have investigated the effect of prolonged androgen therapy on ovarian histology. One study of 112 transgender men who underwent hysterectomy with bilateral oophorectomy after testosterone therapy revealed a polycystic ovarian morphology, such as collagenization of the outer cortex, stromal hyperplasia, luteinization of stroma cells, and multifollicular ovaries (26). In contrast, another study of 40 transgender men on testosterone for more than one year showed preservation of normal cortical follicle distribution (15).

\section{Effects of estrogen therapy}

In transwomen, estrogen therapy suppresses gonadotropin levels, leading to reduced testosterone levels, which can affect spermatogenesis. The effect of GAHT on testicular morphology in transwomen who underwent orchiectomy has been described in 11 studies with variable hormonal treatment regimens (27). Similar to the studies on the effect of testosterone therapy in transmen, these studies showed conflicting results. Outcomes ranged from complete cessation of spermatogenesis with testicular atrophy, hyalinization, and fibrosis to preserved spermatogenesis with normal testicular histology (27-31). Outcomes of one study by Schneider et al. were heterogeneous irrespective of whether patients discontinued hormones 6 weeks before orchiectomy, 2 weeks before, or not at all, with $24 \%$ of patients demonstrating normal spermatogenesis, while the rest had varying degrees of impairment, such as meiotic arrest or spermatogonial arrest (30). Revirilization occurred quickly in the patients who had stopped hormones (30). In another study that evaluated the effect of duration of hormone therapy, there was a trend toward worse spermatogenesis with longer therapies, but this was not statistically significant (31).

In the first study to describe the impact of GAHT on semen parameters, Adeleye et al. evaluated a cohort of 28 transwomen who presented for sperm cryopreservation (32). The authors compared 18 patients who had never used hormones, 3 who had discontinued hormones before specimen collection (mean discontinuation period of 4.4 months), and 7 who had continued hormones at the time of specimen collection. There were significant differences in concentration, motility, and total motile sperm count between the three groups; the hormone-naïve patients had the best semen parameters. Three of the patients who had continued hormones were azoospermic while all patients 
who had discontinued hormones had semen analysis parameters that were within normal limits based on World Health Organization (WHO) reference values (32).

\section{Fertility preservation in prepubertal transgender youth}

Maturation of oocytes or sperm occurs during puberty, but for prepubertal individuals who opt for FP prior to initiating hormonal therapy, options are more limited since they do not have mature gametes to preserve. FP options in these individuals are still investigational; accordingly, these procedures should only be performed under an institutional review board-approved protocol at an expert center (21).

\section{Ovarian tissue cryopreservation}

For prepubertal FTM transgender individuals, the only option for FP is ovarian tissue cryopreservation (OTC). OTC with subsequent ovarian autotransplantation is currently experimental and has been performed primarily in prepubertal girls who require gonadotoxic cancer treatments (33-36). Ovarian tissue obtained via biopsy or oophorectomy is cryopreserved. When patients opt to restore fertility, the tissue is thawed and autotransplanted. If an ovary is still present, the tissue can either be fixed to the decorticated medulla (34) or inserted under the cortex (35). If bilateral oophorectomy was performed, then the ovarian tissue is placed in a peritoneal window (37). If primordial follicles were present, ovarian activity was restored in $100 \%$ of cases (33). At least 60 live births worldwide have been reported using this technique (33). In a study of 20 women who underwent OTC with ovarian autotransplantation, $53 \%$ conceived and $32 \%$ had at least one live birth (35). There has been one documented live birth following autotransplantation of ovarian tissue cryopreserved before menarche (36). Orthotopic ovarian tissue reimplantation allows for the possibility of restoration of fertility and natural conception. However, for FTM individuals, restoration of ovarian function may be undesirable given that it may have feminizing effects.

An alternative method of using the preserved ovarian tissue that does not require restoration of ovarian function is to perform in vitro follicle growth and oocyte maturation (38). The follicles from the ovarian tissue are grown in vitro using a special culture medium with alginate hydrogels. Several studies showed the success of in vitro maturation of oocytes to metaphase II, which demonstrated that this is a promising method for FP (38). If oocytes can successfully mature in vitro, they can potentially be used for in vitro fertilization/intracytoplasmic sperm injection (IVF/ICSI) using either a gestational surrogate or in an FTM individual with a preserved uterus.

\section{Cryopreservation of testicular tissue}

Prepubertal MTF transgender individuals cannot bank sperm, but a potential method of FP is cryopreservation of immature testicular tissue (ITT), which has been performed under experimental protocols. ITT contains spermatogonial stem cells (SSCs) from which spermatozoa derive. Controlled slow-freezing with dimethyl sulfoxide (DMSO) has been used to preserve ITT and protect the tissue architecture (39). This process allows survival and proliferation of spermatogonia after transplantation of isolated testicular stem cells to germ cell-depleted testes in mice (39). Vitrification is a potential alternative technique that uses high concentrations of cryoprotectant and ultrafast cooling speeds to minimize cellular damage in the long-term storage of ITT (40). In one study, spermatogonia were able to survive and proliferate, but only partial differentiation occurred after vitrification and xenotransplantation (40). Another study showed that long-term propagation of SSCs could occur in vitro in a special culture medium (41). Further studies are necessary to improve the ability of SSCs to differentiate, but this is a promising technology that could potentially be used in prepubertal transgender individuals in the future.

\section{Fertility options for transgender men}

\section{Cryopreservation of oocytes or embryos}

For post-pubertal transmen who have not undergone oophorectomy, currently available options for FP include oocyte and embryo banking, both of which require hormonal stimulation for egg retrieval and assisted reproductive technology (ART) for future conception. Embryo banking would require sperm from a donor or partner for IVF/ICSI and could allow for preimplantation genetic diagnosis (PGD) or screening (PGS) prior to cryopreservation. Hormonal stimulation of the ovaries for oocyte retrieval lasts for at least 2 weeks and will lead to increased estrogen, which may exacerbate gender dysphoria for some individuals and become a barrier to FP. After cryopreservation, ART can be done with the transgender 
individual if the uterus has been preserved, or with a gestational surrogate.

In a case report of 3 transgender men who underwent oocyte cryopreservation before starting GAHT, 1 patient was an adolescent while the 2 adults both had partners that carried pregnancies with the preserved oocytes (42). Some transmen who undergo cryopreservation of oocytes experience hesitation and anxiety over having to undergo pelvic and transvaginal examinations (43). There are also physical and mental challenges to dealing with the effects of testosterone cessation such as fatigue, changes in voice and odor, and resumption of menstruation, which can make them feel like they are becoming more feminine, and thus, increase their gender dysphoria (43). These results highlight the importance of healthcare provider sensitivity when working with this vulnerable population.

A potential future option for transgender men that would avoid having to undergo ovarian stimulation is to preserve the ovarian tissue following oophorectomy. As mentioned previously, some studies have shown preserved ovarian histology following prolonged androgen therapy (15). A large number of cumulus-oocyte complexes (COC) can be retrieved from the oophorectomy specimens (15). If these COCs can then be activated and matured in vitro, then transgender men would be able to have children through IVF without having to ever stop GAHT $(38,44)$.

\section{Uterus preservation}

For transmen who undergo GAS that includes hysterectomy or genital reconstruction with vaginal occlusion, gestational pregnancy is no longer a possibility. However, most transmen have not undergone GAS and retain their female reproductive organs, which allows for gestational pregnancy as a family-building option $(4,45,46)$. No studies have evaluated the incidence of pregnancy in transmen, but it is well documented that they can experience planned and unplanned pregnancy during and after GAHT (8-10). In a survey of 197 transgender men, 60 pregnancies were reported, $10(17 \%)$ of which occurred after stopping testosterone and $1(1.6 \%)$ while taking testosterone regularly (8). 30 participants erroneously believed that testosterone was a form of contraception (8). It is currently unclear what sort of effects low dose hormone from contraception can have on exogenous testosterone use in transmen.

In another study of 41 transmen who had been pregnant or delivered after transitioning, the majority had intentional pregnancies $(28,68 \%)$, used testosterone before pregnancy $(25,61 \%)$, and used their own oocytes (36, $88 \%$ ) (9). Twenty resumed menstruation within 6 months of testosterone cessation, while 5 conceived while still amenorrheic. Pregnancy, delivery, and birth outcomes did not differ based on prior testosterone use, though the effect of prior testosterone use on obstetrical complications has not been thoroughly investigated. About half of the men (21, $51 \%)$ chest (breast) fed, but were less likely to do so if they had previously used testosterone (9). Transgender men have strong preferences for either a cesarean section (i.e., to avoid having genitals exposed during birth) or vaginal birth (i.e., because it was an emotionally meaningful experience) (47).

Transmen who have children through pregnancy often feel isolated by the lack of support and resources available to them $(9,47)$. Some individuals feel comfortable in their bodies during pregnancy, while others report that it worsened their gender dysphoria $(9,46)$. Many have negative experiences with healthcare providers and staff $(10,46)$, which could be a reason why they are more likely to use non-physician providers and non-hospital birth locations than the general public (9).

\section{Fertility options for transgender women}

\section{Cryopreservation of sperm or embryos}

Transwomen who have not undergone orchiectomy can utilize sperm banking by providing a semen specimen through masturbation. From a psychological perspective, some individuals find it impossible to masturbate in order to produce a specimen (13). Accordingly, testicular sperm extraction is a potential option, as it is for transgender women who may have fertility problems, such as ejaculatory disorders or azoospermia. Embryo banking would require an oocyte from a donor or partner for IVF/ICSI. Although sperm cryopreservation is ideal before initiating GAHT, new data show that within months of discontinuation of GAHT, some transgender women may produce a semen specimen with sperm concentration and motility adequate for intrauterine insemination (IUI) or spontaneous conception (32).

The largest study evaluating the incidence of sperm banking and semen quality among transwomen revealed an increasing incidence of sperm cryopreservation in this population (48). Surprisingly, compared to cisgender sperm bankers, the 78 transwomen included in the study had worse semen parameters, including sperm concentration 
and total motile sperm count (48). Similarly, another study showed high rates of oligozoospermia, asthenozoospermia, and teratozoospermia, though there was no control group (49). Possible explanations of the worse semen parameters could be that hormonal therapy in these patients was not reported, or there could be a confounding variable that is disproportionately affecting the spermatogenesis of transwomen compared to ciswomen. For instance, many transwomen engage in "tucking", a term used to describe the hiding of testicles and the penis (50). The testicles may be pushed into the inguinal canal or compressed between the legs, which could create a suboptimal environment for spermatogenesis.

\section{Uterus transplantation}

More than 42 uterus transplantations have been performed around the world and at least 12 live births have been reported (51). The first uterus transplant was performed in 2002 in Saudi Arabia using a live donor, but the graft failed several months later and had to be removed (52). In 2011, the first cadaveric uterus transplant and first pregnancy occurred in Turkey, though the pregnancy was not successful (53). In 2014, Swedish physicians reported the first successful live birth of a child from a uterus transplant, and they also carried out several living motherto-daughter uterus transplants $(54,55)$. In 2015, a team in China used a robotic approach for the donor hysterectomy and successfully used ovarian veins rather than uterine veins for venous drainage, significantly reducing the duration of the operation (56). The first live birth after a uterus transplant in the United States occurred in 2016 (57). That same year, researchers from Sweden and Japan were able to bioengineer uterine tissue that could support pregnancy in a rat model (58).

It is clear that uterus transplantation is a rapidly developing field, so it is likely that this treatment option will become more available in the future. The ASRM acknowledged uterus transplantation as the first successful medical treatment of absolute uterus factor infertility (59), but it has not yet been performed in a transgender woman. The Montreal Criteria for the Ethical Feasibility of Uterine Transplantation, which was developed in 2012 to guide physicians and researchers in ethically carrying out uterus transplants, require that the recipient be a cisgender female due to safety and efficacy concerns (60). Animal and human trials have only been performed in cisgender females and there are concerns about creating adequate vasculature for the uterus in a transgender female, placement of the uterus in a non-gynecoid pelvis, and the hormones needed to sustain pregnancy (60). Some transwomen may have the desire to gestate because it aligns with their gender identity (61). Uterus transplantation offers the same solution for transwomen who wish to get pregnant and avoid the use of a gestational carrier as it does for cisgender women with uterus factor infertility (62). Jones et al. argue that uterus transplantation in transwomen may be ethically and legally permissible based on considerations of justice and equality (51).

Transmen could be potential donors for uterus transplant in transwomen. When transmen undergoing hysterectomy and bilateral salpingo-oophorectomy for GAS were asked about their attitude towards uterus donation, $84 \%$ wanted to volunteer for donation (63).

\section{Barriers to care}

Transgender individuals face many obstacles when it comes to accessing healthcare $(4,45)$. Historically, patients who deviate from the norm of the heterosexual, cisgender population have been denied access to ART (6). In some countries, cryopreservation is not available to the transgender population (64). In places where FP is available, there is resistance from some clinicians in providing those services to transgender individuals based on personal biases (65). In the first study that evaluated the experiences of transgender people who sought or accessed assisted reproductive services to have biologically-related children, James-Abra et al. found that 7 of 11 people interviewed had negative experiences with the provider (66). Transgender individuals face discrimination in essentially every aspect of society, including the healthcare setting (67). Some examples include being mis-gendered or refused services altogether solely based on gender identity $(9,10,66)$. In the 2015 U.S. Transgender Survey, 23\% of respondents did not see a doctor when they needed to because of fear of being mistreated (4).

For those who may be interested in fertility services, another barrier is the lack of information (68). A crosssectional survey of the websites of all fertility clinics ( $n=379)$ listed on the Society for Assisted Reproductive Technology (SART) database showed that $53 \%$ contained LGBTQ content, and only $32 \%$ included information for transgender individuals (69). Clinicians need to provide the appropriate information that is specific to this population, but many do not have the education or expertise to adequately counsel patients. The WPATH Standards of Care is based on 
expert opinion and can guide physicians providing FP and reproductive care to transgender individuals, but ultimately, much of the burden of medical decision-making will be placed on the providers (3).

Some individuals lack access due to a scarcity of FP centers (21). Even those who have access to a center may not be able to get the care they seek due to the costs (68). FP can be expensive, with overall costs compounded by the costs associated with gender transitioning, such as GAHT and GAS. There are fees for the consultations, hormones to stimulate oocyte production for transmen, harvesting tissue, tissue processing, the retrieval of gametes, shipment of the specimens, and long-term storage. On top of the costs of FP, there are the costs of ART to consider when a decision is made to use preserved gametes to have children, especially if a gestational surrogate is needed. Insurance coverage for these services remains limited. Many transgender individuals lack social and/or financial support from family and friends, which creates another obstacle (68).

In addition to financial costs, there can be an emotional cost to FP. Transmen who need to take hormones for oocyte harvesting and transwomen who need to masturbate to provide a semen sample may experience distress and worsening gender dysphoria $(13,43,68)$. Some transmen may find it emotionally challenging to preserve their uterus or to gestate given its association with a female identity $(9,46)$.

\section{Ethical concerns}

Historically, a central ethical concern has been whether it is acceptable to help transgender individuals reproduce (2). It has been argued that transgender people are mentally unfit to parent, and thus, should be denied access to reproductive services (65). In fact, there are sterilization provisions in many countries that are promoted under the argument of protecting child welfare (7). Twenty countries in Europe continue to enforce a sterilization requirement for transgender individuals who apply for gender recognition, which forces them to either give up their reproductive capabilities or surrender their right to be acknowledged by their preferred gender (7). The argument that trans people are not fit to be parents is outdated, discriminatory, and not evidence based (2). Studies on the children of transgender parents have found no effect on the gender identity or sexual orientation of the children of trans people, nor is there any evidence that the well-being of the children is compromised in any way $(14,61)$.

There are several other ethical issues as well. First of all, the risks of long-term exposure to hormones by transgender individuals is not understood, and thus, any potential risks to the patient or future offspring is unknown. Also, offering any services that are currently experimental could cause harm to the patient, given that they are only available under a research protocol and the risks are still unclear.

In the pediatric population, there are ethical issues regarding the patient's ability to participate in medical decision-making, especially when they are asked to make potentially irreversible fertility decisions as a minor (21). It can also be challenging when the desires of the parents and child are different. From the provider's standpoint, it is also important to set realistic expectations with the patient and family given that the options for FP in prepubertal children are experimental (21). Prepubertal transgender children may be forced to choose whether they want to experience permanent changes to their body associated with puberty or whether they want to transition and risk irreversible infertility (64). The ASRM recommends that any decisions regarding gonadectomy be delayed until the individual is an adult (2).

\section{Future directions}

A growing number of urologists is taking care of transgender patients, and these physicians may be tasked with managing hormonal therapy, GAS, and fertility considerations. As technology advances to provide transgender individuals the ability to have biologically-related children, it is equally important to make advancements in provider and patient education (70). A Gender and Sex Diversity Fertility Group with 12 providers at 8 institutions concluded that a provider assessment tool to assess patients' knowledge and feelings about FP was necessary (70). A design aid would also be helpful to manage complex information and provide unbiased information to patients (70). It is clear that there is a need for more fertility specialists that are educated about and sensitive to the specific needs of the transgender population.

More research is needed to investigate the feasibility of experimental FP options, such as cryopreservation and in vitro maturation of ovarian and testicular tissue and uterus transplantation. It is also necessary to explore the specific needs of transgender youth and adults to better understand their fertility desires and determine the best protocol for fertility counseling. In order to provide better evidencebased information on the medical and psychosocial risks to the patient and offspring, further investigation into the outcomes of FP is critical. 


\section{Conclusions}

Transgender individuals have experienced pervasive discrimination, but they have recently started to make significant gains in societal acceptance. As access to healthcare improves for this patient population, it is important for urologists and the rest of the medical community to address the specific needs of this community and improve the patient experience. Transgender individuals may express the desire to have biological children and should be educated on the FP options available to them, including oocyte cryopreservation and uterus preservation for transmen and sperm cryopreservation for transwomen. Experimental and future options may include cryopreservation of ovarian or testicular tissue for prepubertal transgender youth and uterus transplantation for transwomen. Further research is needed to explore the outcomes of these options and improve access to and quality of care.

\section{Acknowledgments}

None.

\section{Footnote}

Conflicts of Interest: The authors have no conflicts of interest to declare.

\section{References}

1. Flores AR, Herman JL, Gates GJ, et al. How many adults identify as transgender in the United States? Los Angeles, California: The Williams Institute, 2016.

2. Ethics Committee of the American Society for Reproductive Medicine. Access to fertility services by transgender persons: An Ethics Committee opinion. Fertil Steril 2015;104:1111-5.

3. Coleman E, Bockting W, Botzer M, et al. Standards of care for the health of transsexual, transgender, and gendernonconforming people, version 7. Int J Transgenderism 2011;13:165-232.

4. James S, Herman J, Rankin S, et al. The report of the 2015 U.S. transgender survey. Washington, DC: National Center for Transgender Equality, 2016.

5. Hembree WC, Cohen-Kettenis PT, Gooren L, et al. Endocrine treatment of gender-dysphoric/genderincongruent persons: an Endocrine Society clinical practice guideline. Endocr Pract 2017;23:1437.
6. De Wert G, Dondorp W, Shenfield F, et al. ESHRE Task Force on Ethics and Law 23: medically assisted reproduction in singles, lesbian and gay couples, and transsexual people. Hum Reprod 2014;29:1859-65.

7. Dunne P. Transgender sterilisation requirements in Europe. Med Law Rev 2017;25:554-81.

8. Light A, Wang LF, Zeymo A, et al. Family planning and contraception use in transgender men. Contraception 2018;98:266-9.

9. Light AD, Obedin-Maliver J, Sevelius JM, et al. Transgender men who experienced pregnancy after female-to-male gender transitioning. Obstet Gynecol 2014;124:1120-7.

10. Hoffkling A, Obedin-Maliver J, Sevelius J. From erasure to opportunity: a qualitative study of the experiences of transgender men around pregnancy and recommendations for providers. BMC Pregnancy Childbirth 2017;17:332.

11. Tornello SL, Bos H. Parenting intentions among transgender individuals. LGBT Health 2017;4:115-20.

12. Wierckx K, Van Caenegem E, Pennings G, et al. Reproductive wish in transsexual men. Hum Reprod 2012;27:483-7.

13. De Sutter P, Kira K, Verschoor A, et al. The desire to have children and the preservation of fertility in transsexual women: a survey. Int J Transgenderism 2002;6:215-21.

14. Stotzer RL, Herman JL, Hasenbush A. Transgender parenting: a review of existing research. In: Institute TW, editor. Los Angeles, CA, 2014.

15. De Roo C, Lierman S, Tilleman K, et al. Ovarian tissue cryopreservation in female-to-male transgender people: insights into ovarian histology and physiology after prolonged androgen treatment. Reprod Biomed Online 2017;34:557-66.

16. Nahata L, Tishelman AC, Caltabellotta NM, et al. Low fertility preservation utilization among transgender youth. J Adolesc Health 2017;61:40-4.

17. Russell AM, Galvin KM, Harper MM, et al. A comparison of heterosexual and LGBTQ cancer survivors' outlooks on relationships, family building, possible infertility, and patient-doctor fertility risk communication. J Cancer Surviv 2016;10:943.

18. Chen D, Matson M, Macapagal K, et al. Attitudes toward fertility and reproductive health among transgender and gender-nonconforming adolescents. J Adolesc Health 2018;63:62-8.

19. Chen D, Simons L, Johnson EK, et al. Fertility preservation for transgender adolescents. J Adolesc Health 2017;61:120-3. 
20. Hembree WC. Guidelines for pubertal suspension and gender reassignment for transgender adolescents. Child Adolesc Psychiatr Clin N Am 2011;20:725-32.

21. Finlayson C, Johnson EK, Chen D, et al. Proceedings of the working group session on fertility preservation for individuals with gender and sex diversity. Transgend Health 2016;1:99-107.

22. Johnson EK, Finlayson C. Preservation of fertility potential for gender and sex diverse individuals. Transgend Health 2016;1:41-4.

23. Hagen CP, Sorensen K, Anderson RA, et al. Serum levels of antimullerian hormone in early maturing girls before, during, and after suppression with $\mathrm{GnRH}$ agonist. Fertil Steril 2012;98:1326-30.

24. Schagen SE, Cohen-Kettenis PT, Delemarre-van de Waal HA, et al. Efficacy and safety of gonadotropin-releasing hormone agonist treatment to suppress puberty in gender dysphoric adolescents. J Sex Med 2016;13:1125-32 .

25. Pasquino AM, Pucarelli I, Accardo F, et al. Long-term observation of 87 girls with idiopathic central precocious puberty treated with gonadotropin-releasing hormone analogs: impact on adult height, body mass index, bone mineral content, and reproductive function. J Clin Endocrinol Metab 2008;93:190-5.

26. Grynberg M, Fanchin R, Dubost G, et al. Histology of genital tract and breast tissue after long-term testosterone administration in a female-to-male transsexual population. Reprod Biomed Online 2010;20:553-8.

27. Schneider F, Kliesch S, Schlatt S, et al. Andrology of maleto-female transsexuals: influence of cross-sex hormone therapy on testicular function. Andrology 2017;5:873-80.

28. Thiagaraj D, Gunasegaram R, Loganath A, et al. Histopathology of the testes from male transsexuals on oestrogen therapy. Ann Acad Med Singapore 1987;16:347-8.

29. Rodriguez-Rigau LJ, Tcholakian RK, Smith KD, et al. In vitro steroid metabolic studies in human testes. II: Metabolism of cholesterol, pregnenolone, progesterone, androstenedione and testosterone by testes of an estrogentreated man. Steroids 1977;30:729-39.

30. Schneider F, Neuhaus N, Wistuba J, et al. Testicular functions and clinical characterization of patients with gender dysphoria (GD) undergoing sex reassignment surgery (SRS). J Sex Med 2015;12:2190-200.

31. Kent MA, Winoker JS, Grotas AB. Effects of feminizing hormones on sperm production and malignant changes: microscopic examination of post orchiectomy specimens in transwomen. Urology 2018;121:93-6.
32. Adeleye AJ, Reid G, Kao CN, et al. Semen Parameters Among Transgender Women With a History of Hormonal Treatment. Urology 2019;124:136-41.

33. Donnez J, Dolmans MM. Ovarian cortex transplantation: 60 reported live births brings the success and worldwide expansion of the technique towards routine clinical practice. J Assist Reprod Genet 2015;32:1167-70.

34. Donnez J, Dolmans MM. Fertility preservation in women. Nat Rev Endocrinol 2013;9:735-49.

35. Meirow D, Ra'anani H, Shapira M, et al. Transplantations of frozen-thawed ovarian tissue demonstrate high reproductive performance and the need to revise restrictive criteria. Fertil Steril 2016;106:467-74.

36. Demeestere I, Simon P, Dedeken L, et al. Live birth after autograft of ovarian tissue cryopreserved during childhood. Hum Reprod 2015;30:2107-9.

37. Donnez J, Dolmans MM, Demylle D, et al. Livebirth after orthotopic transplantation of cryopreserved ovarian tissue. Lancet 2004;364:1405-10.

38. Xiao S, Zhang J, Romero MM, et al. In vitro follicle growth supports human oocyte meiotic maturation. Sci Rep 2015;5:17323.

39. Wyns C, Curaba M, Vanabelle B, et al. Options for fertility preservation in prepubertal boys. Hum Reprod Update 2010;16:312-28.

40. Poels J, Van Langendonckt A, Many MC, et al. Vitrification preserves proliferation capacity in human spermatogonia. Hum Reprod 2013;28:578-89.

41. Sadri-Ardekani H, Mizrak SC, van Daalen SK, et al. Propagation of human spermatogonial stem cells in vitro. JAMA 2009;302:2127-34.

42. Maxwell S, Noyes N, Keefe D, et al. Pregnancy outcomes after fertility preservation in transgender men. Obstet Gynecol 2017;129:1031-4.

43. Armuand G, Dhejne C, Olofsson JI, et al. Transgender men's experiences of fertility preservation: a qualitative study. Hum Reprod 2017;32:383-90.

44. Lierman S, Tilleman K, Braeckmans K, et al. Fertility preservation for trans men: frozen-thawed in vitro matured oocytes collected at the time of ovarian tissue processing exhibit normal meiotic spindles. J Assist Reprod Genet 2017;34:1449-56.

45. Grant JM, Mottet LA, Tanis J, et al. National transgender discrimination survey report on health and healthcare. Washington, DC: National Center for Transgender Equality and National Gay and Lesbian Task Force, 2010.

46. Obedin-Maliver J, Makadon HJ. Transgender men and pregnancy. Obstet Med 2016;9:4-8. 
47. Ellis SA, Wojnar DM, Pettinato M. Conception, pregnancy, and birth experiences of male and gender variant gestational parents: it's how we could have a family. J Midwifery Womens Health 2015;60:62-9.

48. Li K, Rodriguez D, Gabrielsen JS, et al. Sperm cryopreservation of transgender individuals: trends and findings in the past decade. Andrology 2018;6:860-4.

49. Hamada A, Kingsberg S, Wierckx K, et al. Semen characteristics of transwomen referred for sperm banking before sex transition: a case series. Andrologia 2015;47:832-8.

50. Conard LE. Supporting and caring for transgender and gender nonconforming youth in the urology practice. $J$ Pediatr Urol 2017;13:300-4.

51. Jones BP, Williams NJ, Saso S, et al. Uterine transplantation in transgender women. BJOG 2019;126:152-6.

52. Fageeh W, Raffa H, Jabbad H, et al. Transplantation of the human uterus. Int J Gynaecol Obstet 2002;76:245-51.

53. Ozkan O, Akar ME, Erdogan O, et al. Uterus transplantation from a deceased donor. Fertil Steril 2013;100:e41.

54. Brannstrom M, Johannesson L, Bokstrom H, et al. Livebirth after uterus transplantation. Lancet 2015;385:607-16.

55. Brannstrom M, Johannesson L, Dahm-Kahler P, et al. First clinical uterus transplantation trial: a six-month report. Fertil Steril 2014;101:1228-36.

56. Wei L, Xue T, Tao KS, et al. Modified human uterus transplantation using ovarian veins for venous drainage: the first report of surgically successful robotic-assisted uterus procurement and follow-up for 12 months. Fertil Steril 2017;108:346-56.e1.

57. Testa G, McKenna GJ, Gunby RT Jr, et al. First live birth after uterus transplantation in the United States. Am J Transplant 2018;18:1270-4.

58. Hellstrom M, Moreno-Moya JM, Bandstein S, et al. Bioengineered uterine tissue supports pregnancy in a rat model. Fertil Steril 2016;106:487-96.e1.

Cite this article as: Cheng PJ, Pastuszak AW, Myers JB, Goodwin IA, Hotaling JM. Fertility concerns of the transgender patient. Transl Androl Urol 2019;8(3):209-218. doi: 10.21037/ tau.2019.05.09
59. Practice Committee of the American Society for Reproductive Medicine. American Society for Reproductive Medicine position statement on uterus transplantation: a committee opinion. Fertil Steril 2018;110:605-10.

60. Lefkowitz A, Edwards M, Balayla J. The Montreal criteria for the ethical feasibility of uterine transplantation. Transpl Int 2012;25:439-47.

61. Murphy TF. Assisted gestation and transgender women. Bioethics 2015;29:389-97.

62. Alghrani A. Uterus transplantation in and beyond cisgender women: revisiting procreative liberty in light of emerging reproductive technologies. J Law Biosci 2018;5:301-28.

63. Api M, Boza A, Ceyhan M. Could the female-to-male transgender population be donor candidates for uterus transplantation? Turk J Obstet Gynecol 2017;14:233-7.

64. Bizic MR, Jeftovic M, Pusica S, et al. Gender dysphoria: bioethical aspects of medical treatment. Biomed Res Int 2018;2018:9652305.

65. Jones HW Jr. Gender reassignment and assisted reproduction. Evaluation of multiple aspects. Hum Reprod 2000; $15: 987$.

66. James-Abra S, Tarasoff LA, Green D, et al. Trans people's experiences with assisted reproduction services: a qualitative study. Hum Reprod 2015;30:1365-74.

67. Rodriguez A, Agardh A, Asamoah BO. Self-reported discrimination in health-care settings based on recognizability as transgender: a cross-sectional study among transgender U.S. citizens. Arch Sex Behav 2018;47:973-85.

68. Mitu K. Transgender reproductive choice and fertility preservation. AMA J Ethics 2016;18:1119-25.

69. Wu HY, Yin O, Monseur B, et al. Lesbian, gay, bisexual, transgender content on reproductive endocrinology and infertility clinic websites. Fertil Steril 2017;108:183-91.

70. Johnson EK, Chen D, Gordon EJ, et al. Fertility-related care for gender and sex diverse individuals: a provider needsassessment survey. Transgend Health 2016;1:197-201. 